\section{Umwelthormone können Allergien fördern}

Endokrine Disruptoren, auch als Xeno- bzw. Umwelthormone bekannt, können zur allergischen Sensibilisierung gegen Aero- und Nahrungsallergene beitragen. Voraussetzung dafür scheint eine antimikrobielle Wirkung zu sein.

\footnotetext{
W issenschaftler aus Baltimore haben den Zusammenhang von endokrinen Disruptoren (EDC) und allergischer Sensibilisierung in einer Studie untersucht, für die sie auf die Daten von 860 Kindern und Jugendlichen (sechs bis 18 Jahre alt) aus den Jahren 2005 und 2006 zurückgegriffen haben. Dabei setzten die Forscher die Urinkonzentrationen von Bisphenol A, Triclosan, Benzophenon 3, Propyl-, Methyl-, Butyl- und Ethyl-Paraben in Beziehung zu den IgE-Spiegeln gegen Aeroallergene (z. B. Birken-, Roggen- und Ambrosiapollen) und Nahrungsallergene (Milch, Eier, Erdnuss, Krabben). Als Grenzwert galten IgE-Konzentrationen von $0,35 \mathrm{kU} / \mathrm{l}$.

Ein Gruppeneffekt der EDC war nicht nachzuweisen. Allerdings stieg die Quote an Sensibilisierungen gegen Aeroallergene
}

signifikant mit den Urinpegeln von Triclosan und Propyl- bzw. Butyl-Paraben (Odds Ratio [OR] des höchsten zum niedrigsten Terzil: 1,51-2,04). Ein statistisch relevanter Zusammenhang mit Nahrungsallergenen ließ sich nur bei Knaben und jungen Männern belegen (OR: 3,9).

Triclosan weist ebenso wie Paraben antimikrobielle Wirkungen auf. Ersteres wird etwa in Zahnpasta, Mundwasser und Mitteln zur Händedesinfektion, letzteres als Konservierungsmittel in Nahrungsmitteln, Medikamenten und Kosmetika verwendet. Das legt es für die Forscher nahe, den sensibilisierenden Effekt nicht mit den endokrinen, sondern mit den antimikrobiellen Eigenschaften der EDC zu verbinden. EDC, die nicht gegen Keime wirken, wie Bisphenyl oder Benzophenon, sind nicht mit Sensibilisierungen assoziiert. Manifeste Erkrankungen wie Asthma ließen sich allerdings nicht mit den EDCSpiegeln korrelieren, auch nicht mit Triclosan und Paraben. Um die potenzielle Rolle antimikrobieller EDC bei allergischen Krankheiten zu klären, sind weitere Studien nötig.

Fazit: EDC-Spiegel im Urin sind mit einer Sensibilisierung gegen Allergene in Luft und Nahrungsmitteln assoziiert - aber nur, wenn sie antimikrobiell wirken. Für nur endokrin wirkende Substanzen lässt sich der Effekt nicht feststellen. Das Ergebnis der vorliegenden Studie weist daher in die gleiche Richtung wie frühere Untersuchungen, die einen Zusammenhang zwischen antibiotisch wirksamen Produkten, dem Mikrobiom und allergischen Erkrankungen hergestellt haben. Dr. Robert Bublak

Savage JH et al. Urinary levels of triclosan and parabens are associated with aeroallergen and food sensitization. J Allergy Clin Immunol 2012; 130: 453-60.e7

\title{
Achtung:
}

\section{Hier muss der Dummy durch eine Anzeige ersetzt werden !!}

